\title{
Imagung Techniques for Medical Applications
}

\author{
Mostafa Ab Ebrahim* \\ Department of Civil Engineering, Assiut University Assiut, Egypt
}

Received: March 21,2018; Published: April 06, 2018

*Corresponding author: Mostafa AB Ebrahim, Assistant Professor Civil Engineering Department Faculty of Engineering, Assiut University Assiut, EGYPT, Tel: +966-553484223; Email: mostafa.ebrahim@btinternet.com

Abbreviations: CT: Computed Tomography; MRI: Magnetic Resonance Imaging; DBT : Digital Breast Tomosynthesis

\section{Introduction}

Modern Engineering Technologies are playing a main roll in medicine treatments. These technologies enable the doctors going under the skins, through the tissues up to the bones. They take them not just deep inside the human bodies, but deeper up to several levels like the womb. They give them the ability to inspect whatever deep inside the human body and give the suitable treatment and solutions for the issues at hand. Of course, without doctors' excellent understanding of the human bodies, nothing can be done because they employing the results of these technologies to understand the case and find the best treatment and solution for it. Modern Engineering Technologies and Medical Application are two main terms of science have a great integration helping people with severe medical issues to find a solution for their medical problems. There are different imaging techniques that being used to show and discover whatever inside the human body. These techniques are called medical imaging. Medicinal imaging is the system and procedure of making visual portrayals of the inside of a body for clinical examination and therapeutic mediation, and in addition visual portrayal of the capacity of a few tissues or organs (physiology). Medical imaging uncover inward structures covered up by the skin and/or bones, and to analyze and treat infection. Medicinal imaging additionally builds up a database of ordinary life structures and physiology to make it conceivable to distinguish variations from the norm. Despite the fact that imaging of expelled organs and tissues can be performed for medical reasons, such methodology are generally considered piece of pathology rather than medicinal imaging.

In present day solution, medical imaging has experienced real headways. Today, this capacity to accomplish data about the human body has numerous valuable clinical applications. Throughout the years, diverse sorts of medical imaging have been produced, each with their own particular focal points and weaknesses. The start was with X-rays imaging which utilizes a
X-beam shaft that is directed to the body. When going through the body, parts of the $x$-beam bar are absorbed. On the contrary side of the body, the X-beams are received, shown as an image. X-ray based techniques for medical imaging incorporates regular X-ray, mammography and computed tomography (CT). To improve the $\mathrm{X}$-ray image, differentiate operators can be utilized for instance for angiography examinations. The most well-known strategies for $\mathrm{X}$-ray in medical imaging are:

a. Radiography (Plain X-rays), called traditional X-rays, have progressed significantly since the soonest radiological imaging and, today, advanced X-ray systems are supplanting film.

b. Computed Tomography (CT) scanners create highly resolution images of the body. Because of their high quality, these images can give extra data contrasted with customary radiography.

c. Mammography is a particular sort of X-ray imaging used to make itemized images of the breast and is regularly utilized as a part of screening for breast cancer growth.

d. Angiography is a particular kind of X-ray strategy for showing veins, arteries and organs, particularly the heart, by inject a colored safe agent into the blood that upgrades its perceivability on the X-ray image.

e. Fluoroscopy is a sort of medical imaging that creates a constant 'live' X-ray image of the patient's inner structures on a screen.

f. Molecular imaging gives itemized data of the organic procedures occurring in the body at cell and molecular levels and can demonstrate ailment in its soonest arranges. Inside the field of molecular imaging, radioactive markers, called radiopharmaceuticals, are utilized to envision the degree of sickness. Consolidated Modalities in molecular imaging 
can be utilized as different imaging advances can be utilized each with its own particular advantages. Different kinds of medical imaging are available such as ultrasound imaging and magnetic resonance imaging (MRI). Dissimilar to ordinary X-ray, ultrasound and MRI work without ionizing radiation. MRI utilizes powerful magnetic fields that create obscure irreversible natural impacts in people.

g. Ultrasound, occasionally known as sonography, is utilized to see fine tissues, for example, muscles; interior organs and blood stream particularly continuously, while MRI conveys magnificent fine tissue stand out from high determination. In Ultrasound imaging, digital images are delivered by sound waves reflected by organs and other inside body parts continuously.

h. Magnetic resonance imaging (MRI) is a complex medical imaging strategy that utilizations magnetic fields and radiofrequency to show the body's inner structures.

The biochemical movement of the cells changes when illness happens and, as it advances, this anomalous action begins to influence the body and cause changes to bones and tissue that won't not be seen by ordinary CT or MRI images. Malignancy cells, for instance, start by duplicating at an expansion rate and after that frame a mass or tumor. By recognizing these progressions sooner, specialists can make medicinal move at a significantly prior phase of the infection than they could already. 3D strategies are considered as a standout amongst the most Modern Engineering methods engaged with the medical applications. 3D methods have wide spreads applications from estimating to printing. Volume rendering systems have been created to empower CT, MRI and ultrasound examining programming to deliver 3D images for the doctor. Customarily CT and MRI checks delivered 2D static yield on film. To create 3D pictures, numerous outputs are made, and afterward joined by PCs to deliver a 3D display, which would then be able to be controlled by the doctor. 3D ultrasounds are created utilizing to some degree comparable strategy. In diagnosing illness of the viscera of abdomen, ultrasound is especially touchy on imaging of biliary tract, urinary tract and female conceptive organs (ovary, fallopian tubes).

3D representation techniques are a profitable asset for the finding and surgical treatment of numerous pathologies. Mammography is a digital breast tomosynthesis (DBT), or 3D mammography, is the energizing new standard in breast imaging because of its spectacular refinement in lesion perceivability and in early cancer recognition. With DBT, a sequence of images are produced for the breast as opposed to joining two projections of images. DBT empowers radiologists to see each tissue layer freely, which decreases the quantity of blunders and the quantity of reviews. 3D Ultrasonic Holography is an innovation picked up footing and is proceeded with its development as of recently. Since ultrasonic holography does not utilize perilous radiation, it is perfect for preventive and post-agent examinations in breast disease patients. The determination of produced images is high in contrast with those of a typical ultrasound. Moreover, the images are effortlessly reproducible and permit computerized PC based information elucidation. 3D printing for medical applications has been soaring. The rundown of items that have just been effectively printed in this field shows the potential that this innovation holds for medicinal services soon, for example, organs, tissues with blood vessels, medical models, bone, tailormade sensors, heart valve, cranium replacement, medical equipment, ear cartilage, synthetic skin, and Low-Cost prosthetic parts.

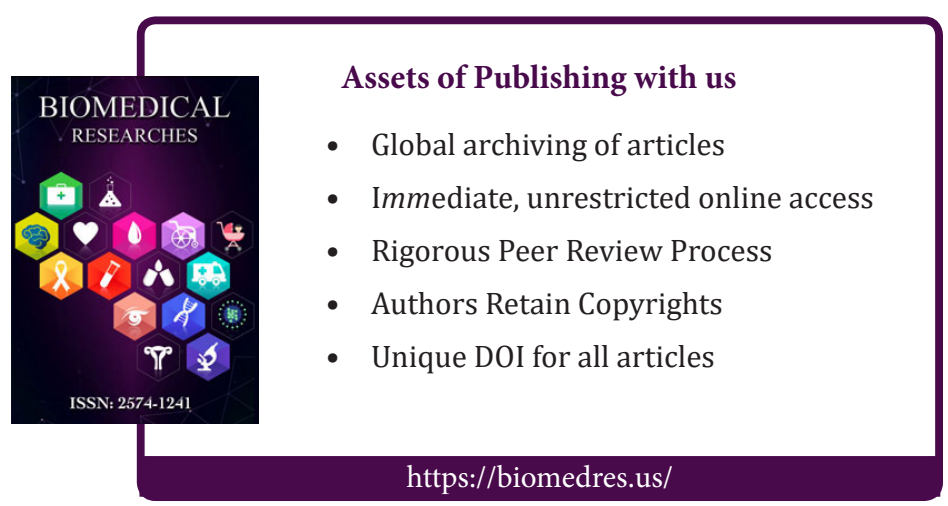

